\title{
Safety Road Travelling System with Connected Vehicles
}

\author{
B. Perumal, E. Naveen Kumar, P. Deepthi, K. Bhavana
}

\begin{abstract}
Now a days we had seen so many Road accident cases are occurring and also increasing day by day. According to the statics of World Health Organization (WHO) 20-50 billion people were losing their life due to these accidents. To avoid these problems we came up with a proposed system called connected vehicles. Vehicle to Vehicle communication is a wireless broadcast that transmits the data between the connected vehicles. The main motive of this connected vehicles is safe travelling without any obstacles between the vehicles. Road accidents are the serious issues for human life for both individuals as well as the economic aspects. So our proposed system "Connected Vehicles" will reduce the accident cases by communicating with the nearer vehicles and shares the necessary information regarding the accidents cases to nearer vehicles.
\end{abstract}

Keywords: Connected Vehicles, Road accidents (key words)

\section{INTRODUCTION}

$\mathrm{E}_{\text {very year a thousand of people losing their life de to }}$ these accident cases. Accident cases are the biggest issues which we are facing now a days. Only in India over 1,50,112 people were killed among 4,21,423 people, an average of 412 people were died per day and per every hour 17 people were losing their lives. So many technologies were implemented to avoid these problems to control the accident cases but still we are facing the same issues. Now a days we had a latest technology implemented in such a way that every moment will sense by many kinds of sensors. By using these kinds of sensors, we proposed a system which works based on the wireless network communication. Wireless communication is the method which helps to connect the vehicles and allows to transmit the data as well as receives the data. By using these network modules wee implement a system called connected vehicles. Connected vehicles is a technology used to avoid the vehicles crashing and provide a safety application for the drivers.

Manuscript received on May 24, 2021.

Revised Manuscript received on May 31, 2021.

Manuscript published on July 30, 2021.

* Correspondence Author

Dr. B. Perumal, Associate Professor, Department of ECE, Kalasalingam Academy of Research and Education, Tamil Nadu-626 126, India. Email: perumal@klu.ac.in

E. Naveen Kumar, Department of ECE, Kalasalingam Academy of Research Research and Education, Tamil Nadu-626 126, India. Email: enaveenkumar111@gmail.com

P. Deepthi, Department of ECE, Kalasalingam Academy of Research Research and Education, Tamil Nadu-626 126, India. Email: pasaladeepu.2018@gmail.com

K. Bhavana*, Department of ECE, Kalasalingam Academy of Research Research and Education, Tamil Nadu-626 126, India. Email: banu.kanthuri04@gmail.com

(C) The Authors. Published by Blue Eyes Intelligence Engineering and Sciences Publication (BEIESP). This is an open access article under the CC BY-NC-ND license (http://creativecommons.org/licenses/by-nc-nd/4.0/)
The communication takes place between the two connected vehicles through wireless network module. The approximate range of this is $900-1000$ meters. This device used 4-5 GHZ band for the communication purpose in Vehicles. These connected vehicles are not only used for communication purpose but also provides tracking the surface of road infrastructure to avoid the crashes. In connected vehicles by using Global Navigation Satellite System (GNSS) we track the Vehicle position and also gather the nearer vehicles position. It also tracks the vehicle speed, location through GPS and accelerometer sensor. While driving a vehicle, crash disclosure plays an critical occupation in independent driving and for the driver. This paper gives an integrative method to bargain speed and course area which expects to be basically pretty much as essential as possible to engage the steady computation while having the choice to change in accordance with an arrangement of road and metropolitan circumstances on different conditions. In this work, we complete a sign based forward sway notice system and a staggering vehicle disclosure structure. The forward crash notice structure expects to perceive various vehicles driving before the host vehicle while overpowering to evade the possible car accidents. Of course, the unparalleled vehicle ID structure is to distinguish various vehicles behind or on the host vehicle to give an alert to the driver when the vehicle begins overpowering. Preliminary outcomes are given real scene. The introduction evaluation has shown the practicality of thefuture strategies.

We are using Arduino uno which is a microcontroller based on the ATMegha328. Arduino uno is flexible for using both the software and the hardware by connecting it through a cable. Arduino Integrated Development Environment is the software part in which we write the programming part in c. Itwrite the Program in Arduino IDE and upload the program in the Arduino board which helps to communicate with each other. Ultrasonic sensor will interface with this Arduino board. Ultrasonic sensor is a device that measures the distance between the vehicles. It provides a safety travel for the drivers without occurring of any obstacles in between the vehicles. Ultrasonic sensors will sense the ultrasound waves. Generally it emits the ultrasonic sound waves and then it get an electrical signal as a reflected waves. The feature of this ultrasonic sensor is, the ultrasonic waves traves faster than the normal audible sound waves. So when two vehicles comer nearer to the another then ultrasonic sensor sense the signal waves and provide the alert messages that a vehicle is approaching nearer to your vehicle. The nRF24Lo1 module is interface with Arduino uno which helps to transmit and receives the data. Two vehicles can communicate the data by using nRF24L01 module.
Blue Eyes Intelligence Engineering and Sciences Publication (C) Copyright: All rights reserved.

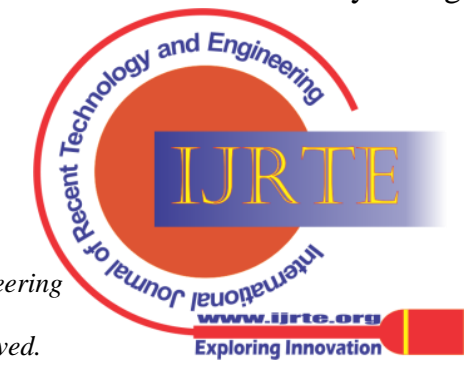


The nRF24L01 is a wireless module which connects the two Arduino boards. When we have more than on e Arduino board nR24fL01 plays an important role which helps to communicate with two or more Arduino boards. It can communicate a long distances in an open source. Accelerometer sensor used to know the speed of the vehicle if the speed is more than the normal exit speed which we given a limit in the coding part then it indicates an alert messages. The ultrasonic sensor is coded in such a way that if any vehicle come nearer to the another vehicle less than $2 \mathrm{~cm}$ then it indicates an alert messages but at the same time if vibrational sensor senses any vibrations then immediately it send the information to the Arduino board. The Arduino board passed the information to the nRF24L01 that send the information to nearer vehicles through GPS and GSM module it send the information to the registered mobile numbers, nearer hospitals.

\section{PREVIOUS WORKS}

Syed Amaar Ahmad, abolfazl Hajisami, Hariharan Krishnan, Farid Ahmed-zaid, Ejsam Moradi-pari they proposed a technique for the international automakes through Dedicated short range communication(DSRC). They focused on the basic safety messages, crash warnings. By using the congestion that includes a lot of remote vehicles that validaate and test any connected vehicles with compliance standards. Such that they can demonstrate the heavy congestion with in the 600ms they can track the vehicle accuracy through that they implemented the vehicle to vehicle communicaiton based on the system congestion and performance[1]. Oyvind Risan, Evtim peytcher proposed a protocol for communication between the vehicles through urban traffic conditions. They proposed a $\mathrm{v} 2 \mathrm{v}$ communication protocol which can be able to find and share the traffic condition statistics in an effective way. This protocol was specially designed to collaborate the traffic conditions to the urban people. This was equipped with a self-conformation and self-directed behaviour. That protocol will make the possibility for the nodes to realize and know the exact location of the areas where specific traffic lights exists. The proposed algorithm only have a least load on wireless network modules[6]. A. Vanitha Kartherine, R. Muthymeenakshi, N. Vallilekha were proposed a prototye for accidents avoidance for an autonomous vehicles. They used a protocol Dedicated short range communication(DSRC). By using RFID along with the WAP, GPS and GSM modules. They also provided services like email, video sharing to communicate with the vehicles and Vehicles-to- Infrastructure. Vehicle to infrastructure provides a communication between the Roadside units(RSUs) and vehicle to vehicle (V2V). They can communicate only through Road side units(RSUs) and combined with GPS and as well as WAP. Through these they are providing a safety messages regarsing accident cases[11].Mohamed el Zorkany, they proposed a system based on the internet of things(IOT) that iot became an essential aspect today form day to day in our life. Based on the Internet of thing(IOT) they implemented a system that prevent the road accident cases by using Road side units(RSUs) through Dedicated short range communication(DSRC) they created a communication between the Vehicles. They focuses mainly on the Intelligent - transportation System(ITS). With the help of
Base station with vehicular adhoc network they created a communication between the Road side Units(RSUs). Through that they can identify the road accident cases and send the alert messages[]. Zhu Nanhao et.al was proposed a system called management energy system for wireless network modules. This remote networks utilizes nRF24L01 module methods for the data transmission and it also receives the data and gather the information and utilizes. At last they concluded that the more number of sensors can send and collect the information through any vehicles by using these nRF24L01 module and send and receives the data from nearer vehicles and passes the information to Arduino Board and as well as receives the data.

\section{PROPOSED SYSTEM}

The proposed system consist of different module which interface with each other such as Arduino uno, ultrasonic sensors, nRF24L01 module, vibrational sensor, GPS and GSM modules which are interfacing with each other.

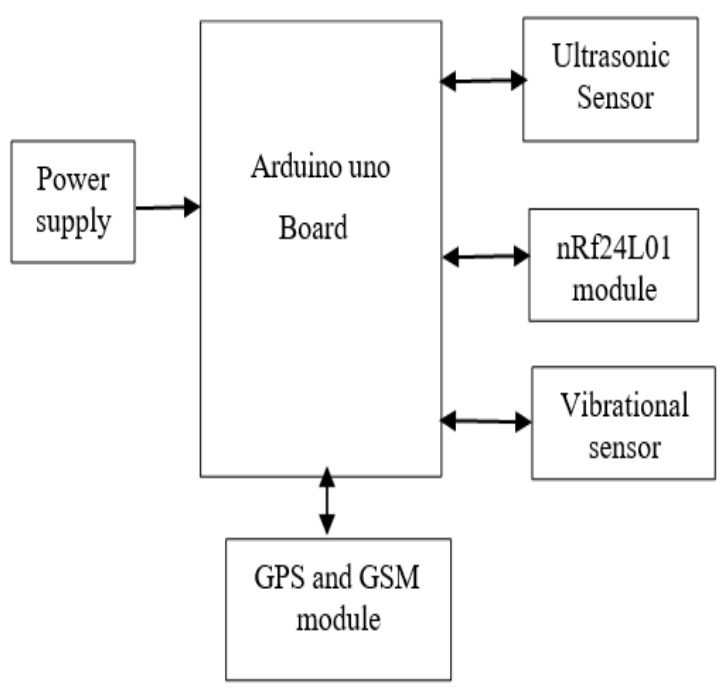

Figure 1. Block diagram of Proposed system

\section{A. Arduino uno}

The Arduino Mega board was related with the distant correspondence module. In all of the vehicles we use Arduino Mega board and far off correspondence module (NRF24L01).It has 8 pins far off module. In the model Arduino Mega board, NRF24L01 distant sensor module, ultrasonic sensor is fixed. Ultrasonic sensor used in the vehicle to stop startlingly when a human or any other obstacles occurs. The essential task of V2V correspondence development is to propel the message between the vehicles. The V2V structure enables correspondence between the vehicles though most of the correspondence is information being imparted starting with one vehicle then onto the next. Each manage certain different signs, for instance, vehicles position, rapidity, need course etc., Arduino integrated development environment is the software part of our proposed system in which the coding is present with embedded c.
Published By:

Blue Eyes Intelligence Engineering and Sciences Publication (C) Copyright: All rights reserved.

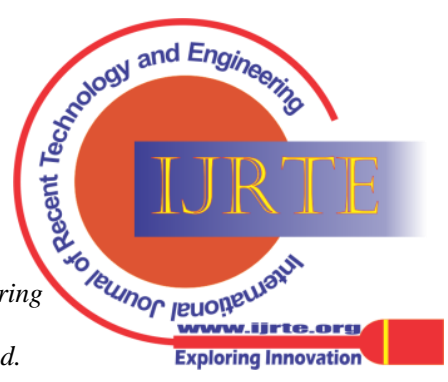




\section{B. Ultrasonic Sensor}

Ultrasonic sensor is a device which is used to measure the distance between two things. It measures the distanceby emitting the ultrasound waves and receives an electrical signal. The range of ultrasonic sensor is $40-70 \mathrm{KHz}$ which is used most widely. It can measures upto $13 \mathrm{feet}(400 \mathrm{~cm})$ range that can sense and measures the distance. In this the ultrasonic sensor interface with Arduino board, when a vehicle approaches nearer to another vehicle less than $2 \mathrm{~cm}$ then it sends an alert message to the host of the vehicle drives that displayed on the LCD. Because the ultrasonic sensor can sense above the human hearing range so that it can detect the obstacle caused by the vehicle drivers easily and sendand alert messages for drivers to maintain a distance between the vehicles.

\section{NRF24LO1 Module}

The nRF24L01 is a wireless chip radio transceiver module which is used to transmit the data as well as receives the data through wireless communication. According to worldwide ISM band its range is 2.4-2.5 GHZ. When we are using more than one Arduino boardit helps to communicate with another Arduino board through that they can share the information easily. In our proposed system these nRF24L01 play a major role to connect the two vehicles. By using these nRF24L01 module we transmit the data and receives the data regarding the accident cases. It will send the messages to nearer vehicles when an accident happens.

\section{Transmitter}

\begin{tabular}{|c|c|}
\hline $\begin{array}{c}\text { Arduino uno } \\
\text { Board }\end{array}$ & $\begin{array}{c}\text { NRF24LO1 } \\
\text { module }\end{array}$ \\
\hline GND & GND \\
\hline Pin No. 3.3volt & VCC \\
\hline Pin No. 9 & CE \\
\hline Pin No. 10 & CSN \\
\hline Pin No. 11 & MOSI \\
\hline Pin No. 12 & MISO \\
\hline Pin No. 13 & SCK \\
\hline
\end{tabular}

Table 1. Arduino uno interfacing with nrf24101 transmitter.

\section{Receiver}

\begin{tabular}{|c|c|}
\hline $\begin{array}{c}\text { Arduino uno } \\
\text { Board }\end{array}$ & $\begin{array}{c}\text { NRF24L01 } \\
\text { module }\end{array}$ \\
\hline GND & GND \\
\hline Pin No. 3.3volt & VCC \\
\hline Pin No. 9 & $C E$ \\
\hline Pin No. 10 & SCN \\
\hline Pin No. 11 & MOSI \\
\hline Pin No. 12 & MISO \\
\hline Pin No. 13 & SCK \\
\hline
\end{tabular}

Table 2. Arduino uno interfacing with nrf24lo1 receiver

\section{GPS AND GSM Module}

Global Positioning System (GPS) is a satellite-based system which provides an exact location. Global System for Mobile Communication (GSM) module is a wireless module network which is used to send and receives the data through a fixed mobile number. Through radio waves it sends and receives the data. By using both GPS and GSM module we can know the exact position of the host vehicle if any sudden accident happens then GSM module will send the information to the registered mobile number regarding the accidents.

\section{E. Vibrating sensor}

Vibrating sensor which is also known as a piezo electric sensor provides an electric signal proportional to acceleration. Accelerometer produces the acceleration then it converts the signal into velocity. Vibrating sensor can sense the fluctuating accelerations. So when an accident takes place it sense the fluctuations. When ultrasonic sensor sense that a vehicle is approaching less than $2 \mathrm{~cm}$ to another vehicle at the same time the vibrating sensor sense the fluctuations then it can confirmed that an accident takes place. Those information sends to Arduino board then Arduino passes information to nRF24L01 as well as GPS and GSM modules then they sent messages to registered mobile numbers and nearby vehicles. Through these the vehiclesconnected to each other.

\section{F. Display output}

In case the two vehicles are pair it shows that whatever rules that the vehicle required feature. It shows the information from our vehicle to the following consolidated vehicle. If are outperforming the vehicle also be appeared on Displacer. It also alerts the host vehicle driver and send messages to registered mobile numbers.

\section{G. Safety Applications}

By using MIT application maker programming we make an .apk archive. Inside the .apk record we arranged left, right, front, back, stop controls. In MIT application planner, we pair an elective which was consolidated to the Bluetooth - This program was made by using a simple C programming. In this application, we can join a device simply far off of 10 meter range. We can add the trap of the web to this suggests, we can deal with our contraption from any spots. When a vehicle came nearer another vehicle then this simple direction will indicated to the host of the vehicle driver through LCD display it like a basic safety messaging system with advanced technology approach for safe driving.

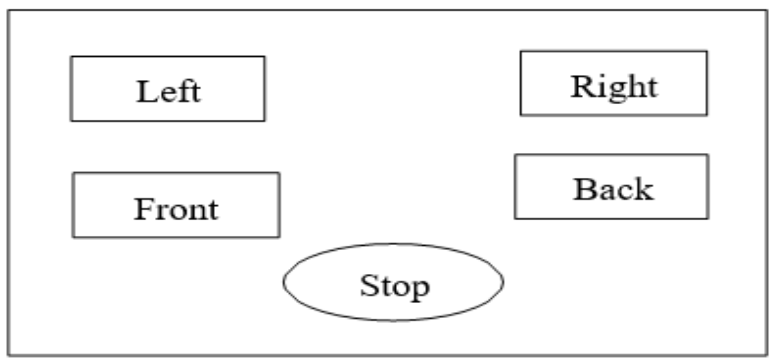

Figure 2. safety message application

Blue Eyes Intelligence Engineering and Sciences Publication

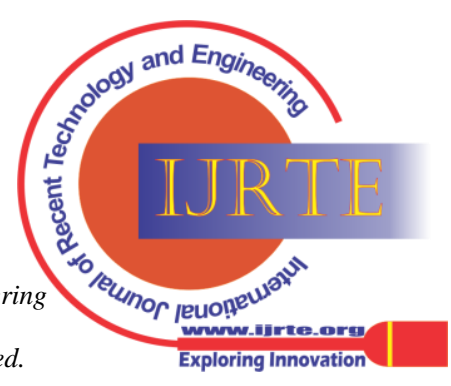




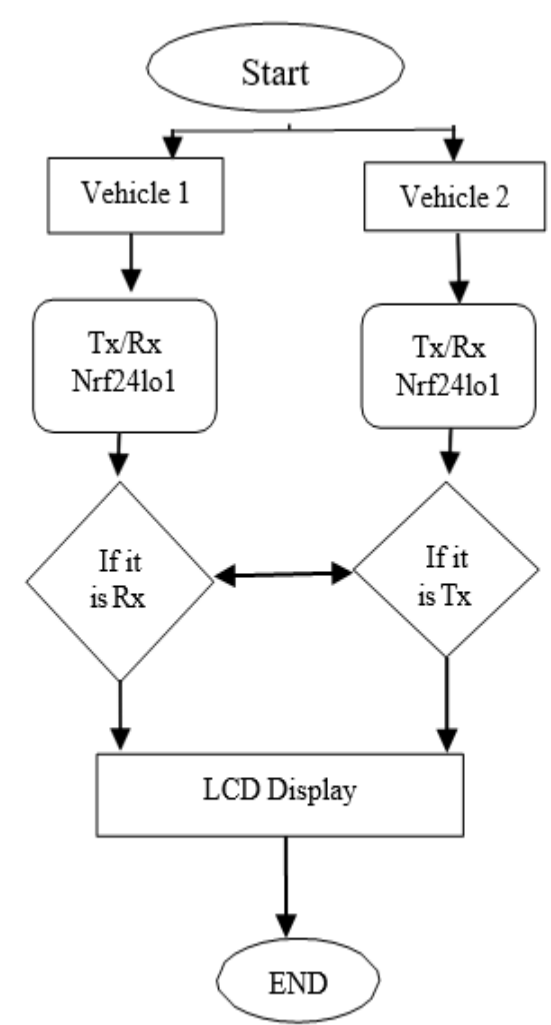

Figure 3. Flow chat for nrf24lo1data transmit and receive

Here we can see the flow chat for nRF24L01 that transmit and receives the data when a particular accident takes place. When a vehicel 1 receives or transmit the information regarding any accidents cased it will display on the LCD screen of the opposite vehcile and also transmit or receives the information regarding the crash issues or accident cases. Finally all the inforamtion gather by the nRF24Lo1and sent the data to Arduino uno and that will send the alert messages to the registered mobile numbers. Through this wireless network modules we will send all the data as well as receives any type of data and can communicate withconnected vehicles.

\section{RESULTS AND DISCUSSION}

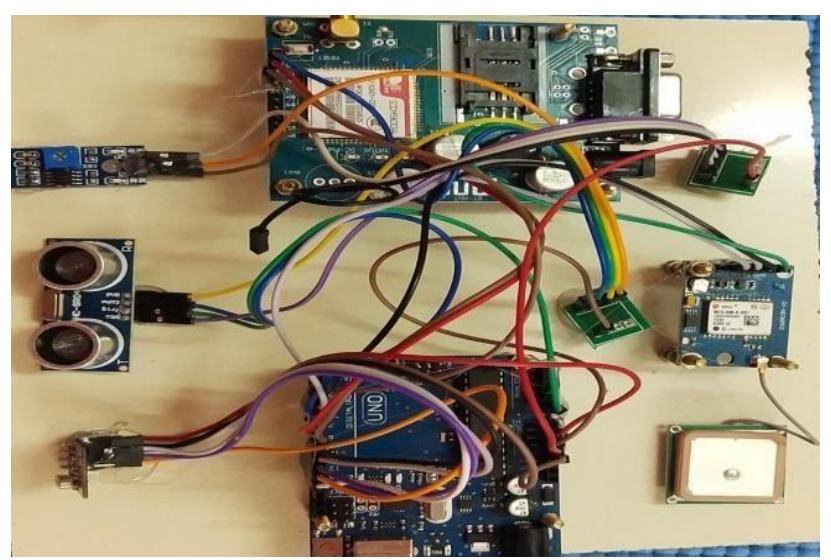

Figure 4. Shows the hardware part of connected vehicle in which Arduino uno interfacing with the ultrasonic sensor, Vibrating sensor, nRF24Lo1 module, GPS and GSM module

This is the prototype of our proposed system that Arduino uno interfacing with the ultrasonic sensor. When an ultrasonic sensor senses the vehicle that present less than $2 \mathrm{~cm}$ to another vehicle at the same time the vibrating sensor sense any vibration then immediately the information sends to Arduino board through nRF24Lo1 network module they transmit or Receives the data regarding the accident issues. By using GPS and GSM module they track the exact position of the vehicle and send the alert messages to register mobile number that they need help and send the information to nearer hospital. This is how our proposed system helps to reduce most of the accident cases.

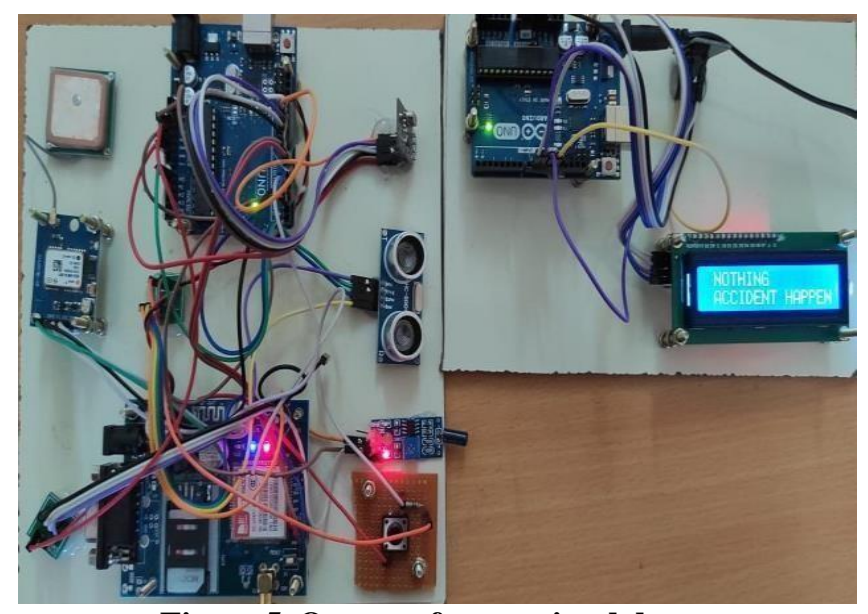

Figure 5. Output of transmitted data.

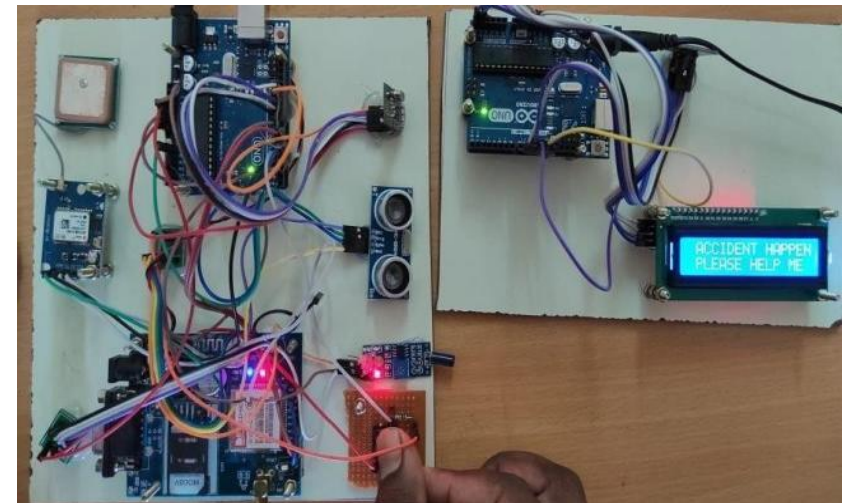

Fig 6. Output for Receiver data when accidents takes place

This is the results of the prototype of the project which shows the emergency alert messages which are connected to the nearer vehicles. Nrf24Lo1 module is used to transmit the data as well as receives the data so when an accident takes place Nrf24lo1 module sends the information to Arduino board that passes the information through nearer vehicles with the help of GSM and GPS module which used to track the exact position of the Vehicle.

When there is no accident it will be in normal state such as it shows nothing accident occurs. When an accident takes places then it indicates Accident happen please help so that the nearer Vehicles can rescue them.

Published By:

Blue Eyes Intelligence Engineering and Sciences Publication

(C) Copyright: All rights reserved.

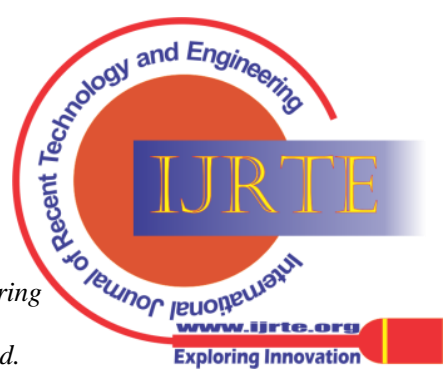




\section{CONCLUSION AND FUTURE WORK}

In this we created a communication between vehicle to vehicle through wireless network modules. It is a set of hardware implementation system that can contact near vehicle and communicated necessary information and gives an alert message when an accident takes place. So, in future it may implemented as a inbuilt function for the cars such that it may easily available for every people to use. And it became a part of the autonomous Vehicles.

\section{ACKNOWLEDGMENT}

The author would like to acknowledge the technical support of guide Dr.B.Perumal sir, Associate Professor in Department of Electronic Communication and Engineering in Kalasalingam Academy of Research and Education. And also we thank for our Kalasalingam University for supporting us on implementation of our project.

\section{REFERENCES}

1. Syed Amaar Ahmad, abolfazl Hajisami, Hariharan Krishnan, Farid Ahmed-zaid, Ejsam Moradi-pari "Vehicle-to-Vehicle Communication for a Platooning System " Transport Research Arena- Europe 2012 by Elsevier. pp1222 - 1233.

2. Yan Zhang, Xuying Zhao, Ri Xu, Jianjin Jiang, and Kejun Zhang " Design and Implementation of a Prototype System for Automatic Obstacle Avoidance Information Collection Vehicle" Springer International Publishing Switzerland 2016.

3. Alfred Daniel1, Anand Paul1, Awais Ahmad, Seungmin Rho " Cooperative Intelligence of Vehicles for Intelligent Transportation Systems (ITS)" School of Computer Science and Engineering, Kyungpook National University, Daegu, Korea.

4. Christian Purucker, Frederik Naujoks , Andy Prill , Alexandra Neukum " Evaluating distraction of in-vehicle information systems while driving by predicting total eyes-off-road times with keystroke level modeling' http://dx.doi.org/10.1016/j.apergo.2016.04.012.

5. Takaya Ezaki, Tomohiro Date, and Hiroyuki Inoue,"An Analysis Platform for the Information Security of In-Vehicle Networks Connected with External Networks" Springer International Publishing Switzerland 2015.

6. Oyvind Risan, Evtim peytcher " A Traffic Light Signal Control Systemwith Truck Priority" PP 377-382 ELSEVIER.

7. Alessandro Bazzi , Alberto Zanella , Barbara M. Masini" A distributed virtual traffic light algorithm exploiting short range

8. Bree Gregorya, Julia D.Irwina, Ian J. Faulksa, Eugene Chekaluk "Differential effects of traffic sign stimuli upon speeding in schoolzones following a traffic light interruption" http://dx.doi.org/ 10.1016/j.aap. 2015.10.020.

9. Katharina Preuk , Eric Stemmler, Meike Jipp " Does surrounding traffic benefit from an assisted driver with traffic light assistance system?" http://dx.doi.org/10.1016/j.trf.2016.09.008.

10. Shaojun Zhang, Ye Wu , Puikei Un , Lixin Fu , Jiming Hao , " Modeling real-world fuel consumption and carbon dioxide emissions with high resolution for light-duty passenger vehicles in a traffic populated city" http://dx.doi.org/10.1016/j.energy.2016.07.067.

11. A.Vanitha Kartherine, R.Muthymeenakshi, N.Vallilekha Towards improving quality of video-based vehicle counting method for traffic flow estimation" http://dx.doi.org/10.1016/j.sigpro.2014.10.035.

12. Wonsik Choi, Dilhara Ranasinghe, Karen Bunavage , J.R. DeShazoc, LisaWuc, Rodrigo Seguel , Arthur M.Winere, Suzanne E. Paulson " The effects of the built environment, traffic patterns, and micrometeorology on street level ultrafine particle concentrations at a block scale: Results from multiple urban sites "http://dx.doi. org/10.1016 /j.scitotenv .2016. 02.083.

13. Ronny Kutadinata, Will Moase, Chris Manzie, Lele Zhang, Tim Garoni "Enhancing the performance of existing urban traffic light controlthroughextremum-seeking" http://dx.doi .org/10.1016/j.trc. V2Vcommunications"http://dx.doi.org/10.1016/j.adhoc.2016.06.006

\subsubsection{6}

14. Hao Chen, Hesham A. Rakha, Amara Loulizi, Ihab El-Shawarby and Mohammed H. Almannaa"Development and Preliminary Field Testing of an In-Vehicle Eco-Speed Control System in the Vicinity of Signalized Intersections", IFAC (International Federation of Automatic Control) Hosting by Elsevier 2016 PP 249-254.

15. Zhenwei Shi, Member, Zhengxia Zou, and Changshui Zhang, Member," Real-Time Traffic Light Detection With Adaptive Background Suppression Filter

"http://10.1109/TITS.2015.2481459.

\section{AUTHORS PROFILE}

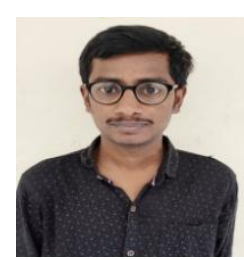

Ekasi Naveen Kumar Reddy, completed Bachelor's of Technology in a stream of Electronic and Communication Engineering in Kalasalingam Academy of Research and Education which is affiliated to Kalasalingam University in Tamil Nadu. I did Safety Road Travelling system with connected vehicles project during my final year and the area of research in VLSI and Embedded systems including Internet of Things, Artificial Intelligence. During research journey i published my journals in IEEE, SCOUPS. And I am currently applied for springer, scopus indexed journals etc. During my project I attended workshops related to Arduino based projects, Artificial Intelligence and got a good experience on it. I also published the projects based on embedded systems in journals.

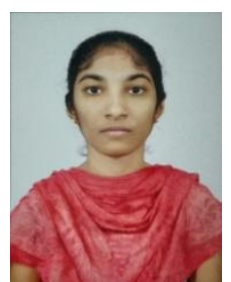

Pasala Deepthi Nagakulayamma, Graduate in Bachelor's of Technology with in specialized stream of Electronics and Communication Engineering. Currently now working on the project based on the domain of Vehicular Communication which has a great demand in the todays world as Safety Road Travelling systems with connected vehicles. As a fresher we are doing projects based on the Technical domain of Arduino based projects. Recently we had completed our Implantation Training on the role of Telecommunication domain on Vizag Steel Plant based on our Research we had gain the information regarding the technical domain and learned a lot of practical knowledge regarding the role of telecommunication field. In my graduation we had researched in the area of Internet of Things and Embedded systems. During my Research I had published in Scopus Journals.

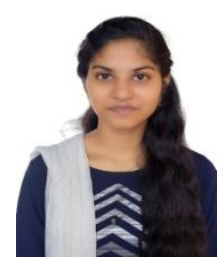

Kanthuri Bhavana, Graduated in Bachelor's of Technology specialized on Electronic and Communication Engineering in Kalasalingam Academy of Research and Education affiliated to Kalasalingam university in Tamil Nadu. Currently working on a project Safety Road Travelling system with connected vehicles. Worked based on the Arduino uno based projects, Internet of things, Embedded systems. I did an implantation training on Vizag Steel plant on a role of Telecommunication which has given a lot of knowledge on wire and wireless technologies. We also published our projects on journals, Scopus indexed journals. I also did a community service project on Agriculture project based on Arduino uno name Torrential Rain Averter and also published on journals.

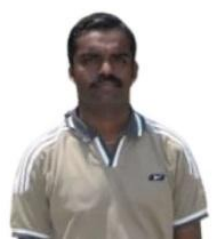

Dr. B. Perumal, M.E., PhD., Associate Professor in Department of Electronic and Communication Engineering in Kalasalingam Academy of Research and Education affiliated to Kalasalingam University in Tamil Nadu. I have been working here since more than 5 years of experience. Currently working on Signals and Systems, Professional Ethics, and Image processing. During my research journey Published so many papers in a reputed journals like IEEE, Scopus. Currently working on Embedded systems, Arduino uno based projects and also worked on the projects based on Antennas and Propagation. Doing Research on new projects based on signals and systems.
Blue Eyes Intelligence Engineering and Sciences Publication (C) Copyright: All rights reserved.

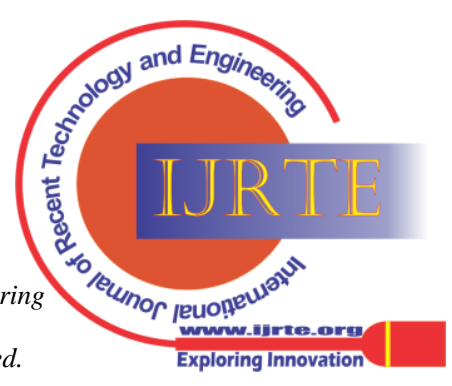

